\title{
HOW MANY ROOTS OF A SYSTEM OF RANDOM TRIGONOMETRIC POLYNOMIALS ARE REAL?
}

\author{
B. KAZARNOVSKII
}

\begin{abstract}
The expected number of zeros of a random real polynomial of degree $N$ asymptotically equals $\frac{2}{\pi} \log N$. On the other hand, the average fraction of real zeros of a random trigonometric polynomial of increasing degree $N$ converges to not 0 but to $1 / \sqrt{3}$. An average number of roots of a system of random trigonometric polynomials in several variables is equal to the mixed volume of some ellipsoids depending on the degrees of polynomials. Comparing this formula with Theorem BKK we prove that the phenomenon of nonzero fraction of real roots remains valid.
\end{abstract}

1. Introduction. For $\lambda=\left(\lambda_{1}, \ldots, \lambda_{n}\right) \in \mathbb{Z}^{n}$ we use the notation $z^{\lambda}=$ $z_{1}^{\lambda_{1}} \cdots z_{n}^{\lambda_{n}}$. Let $\Lambda$ be a finite subset of $\mathbb{Z}^{n}$. We remind that a function $\sum_{\lambda \in \Lambda} a_{\lambda} z^{\lambda}$ on a complex torus $(\mathbb{C} \backslash 0)^{n}$ is called a Laurent polynomial with support $\Lambda$. The convex hull $\operatorname{conv}(\Lambda)$ of $\Lambda$ is called a Newton polytope of Laurent polynomial.

Definition 1. Let the set $\Lambda$ be centrally symmetric. The Laurent polynomial $\sum_{\lambda \in \Lambda} a_{\lambda} z^{\lambda}$ is called a real Laurent polynomial, if

$$
\forall \lambda \in \Lambda: a_{-\lambda}=\overline{a_{\lambda}} .
$$

Any real Laurent polynomial is a real function on the compact subtorus $T^{n}$ of the torus $(\mathbb{C} \backslash 0)^{n}$.

Definition 2. Any real Laurent polynomial with a support $\Lambda$ as a function on $T^{n} \subset(\mathbb{C} \backslash 0)^{n}$ is called a trigonometric polynomial on the torus $T^{n}$ with the support $\Lambda$.

Trigonometric polynomials with support $\Lambda$ form a real vector space $V(\Lambda)$ of dimension equal to the number of points in $\Lambda$. In what follows, we always consider $V(\Lambda)$ as a subspace of the space $L^{2}\left(T^{n}, d \chi\right)$, where $d \chi$ is the normalized Haar measure on the torus $T^{n}$.

Let $l$ be a linear functional in the space $\mathbb{Z}^{n} \otimes_{\mathbb{Z}} \mathbb{R}$. Assume that $l$ is nonzero at nonzero points of the set $\Lambda$. We denote by $\Lambda_{+}$the intersection of $\Lambda$ with the half-space $l \geq 0$. For $0 \neq \lambda \in \Lambda_{+}$we put

$$
\tau_{\lambda}(\theta)=\sqrt{2} \cos \langle\theta, \lambda\rangle, \tau_{-\lambda}(\theta)=\sqrt{2} \sin \langle\theta, \lambda\rangle .
$$

If $0 \in \Lambda$ then we put $\tau_{0}(\theta)=1$. The functions $\left\{\tau_{\lambda}: \lambda \in \Lambda\right\}$ form an orthonormal basis of the space $V(\Lambda)$.

Key words and phrases. trigonometric polynomial, Theorem BKK, mixed volime. 
Zeroes of trigonometric polynomials in one variable have been considered in many publications; see $\mathrm{ADG}$ and the references therein. It turned out that, in contrast to the algebraic case (see [Ka] as well as [EK] and the bibliography therein), the expected fraction of real zeros of a random trigonometric polynomial of increasing degree does not tend to zero. Namely, the following is true. Let $\sum_{0 \leq i \leq m} a_{i} z^{i}+\overline{a_{i}} z^{-i}$ be a random real trigonometric polynomial. Denote by $\mathfrak{M}(m)$ the average number of its zeroes located on the unit circle. Then $\lim _{m \rightarrow \infty} \frac{1}{2 m} \mathfrak{M}(m)=\frac{1}{\sqrt{3}}$; see Example 2 ,

It will be shown below that a similar phenomenon holds true for trigonometric polynomials in several variables. For this we use two theorems on the numbers of roots of systems of equations. The first theorem states that the average number of roots of systems of random trigonometric polynomials $\left\{f_{1}=\ldots=f_{n}=0: f_{i} \in V\left(\Lambda_{i}\right)\right\}$ is equal to the multiplied by $n$ ! mixed volume of some ellipsoids ell $\left(\Lambda_{i}\right)$, depending on the supports $\Lambda_{i}$. This theorem is a specialization of [AK, Theorem 1] to the case $X=T^{n}$ and $V_{i}=V\left(\Lambda_{i}\right)$; see also [ZK]. The second theorem states that for almost all tuples of $n$ real Laurent polynomials with supports $\Lambda_{1}, \ldots, \Lambda_{n}$ the number of their common zeros in the complex torus $(\mathbb{C} \backslash 0)^{n}$ is the same and is equal to the mixed volume of Newton polyhedra $\operatorname{conv}\left(\Lambda_{i}\right)$ multiplied by $n$ !. This statement is a consequence of Theorem BKK; see [B].

2. The average number of roots. Let $\Lambda_{1}, \ldots, \Lambda_{n}$ be finite centrally symmetric sets in $\mathbb{Z}^{n}$, and let $V\left(\Lambda_{i}\right)$ be a space of trigonometric polynomials with the support $\Lambda_{i}, \operatorname{dim} V\left(\Lambda_{i}\right)=N_{i}=\# \Lambda_{i}$. We denote by $S_{i} \subset V\left(\Lambda_{i}\right)$ the sphere of radius 1 centered in 0 . Let $d s_{i}$ be an orthogonally invariant volume form on $S_{i}$, such that $\int_{S_{i}} d s_{i}=1$ (we consider the spaces $V\left(\Lambda_{i}\right)$ with the metric $\left.L^{2}\left(T^{n}, d \chi\right)\right)$. Consider the points $s_{1} \in S_{1}, \ldots, s_{n} \in S_{n}$ as trigonometric polynomials on $T^{n}$, and denote by $N\left(s_{1}, \ldots, s_{n}\right)$ the number of isolated points of the set of their common zeroes.

Definition 3. We call

$$
\mathfrak{M}\left(\Lambda_{1}, \ldots, \Lambda_{n}\right)=\int_{S_{1} \times \ldots \times S_{n}} N\left(s_{1}, \ldots, s_{n}\right) d s_{1} \ldots d s_{n} .
$$

the average number of roots of a system of random trigonometric polynomials with the supports $\Lambda_{1}, \ldots, \Lambda_{n}$.

Let $\Theta_{i}: T^{n} \rightarrow V\left(\Lambda_{i}\right)$ be a map, such that

$$
\forall f \in V\left(\Lambda_{i}\right):\left\langle\Theta_{i}(\theta), f\right\rangle=\frac{1}{\sqrt{N}_{i}} f(\theta) .
$$

Lemma 1. It is true that $\Theta_{i}\left(T^{n}\right) \subset S_{i}$.

Proof. Using the orthonormal basis of the space $V\left(\Lambda_{i}\right)$ defined in (1), we write the mapping $\Theta_{i}$ as

$$
\Theta_{i}(\theta)=\frac{1}{\sqrt{N_{i}}} \sum_{\lambda \in \Lambda} \tau_{\lambda}(\theta) \tau_{\lambda} .
$$


The statement follows from the identity $\cos ^{2}+\sin ^{2}=1$.

Let $\mathfrak{h}$ and $\mathfrak{h}^{*}$ be the tangent and cotangent spaces, respectively, at the zero point of the torus $T^{n}$. We denote by $\mathbb{Z}^{n}$ the integer lattice in the space $\mathfrak{h}$, and by $\mathbb{Z}^{n *}$ - the dual lattice in the space $\mathfrak{h}^{*}$. We further assume that $\Lambda_{i} \subset \mathbb{Z}^{n *}$. Let $F_{i}$ be a quadratic form in $\mathfrak{h}$ which is a pull-back of the metric form on the sphere $S_{i}$ under the mapping $\Theta_{i}$ at the zero point of $T^{n}$.

Lemma 2. It is true that $F_{i}(\xi)=\frac{1}{N_{i}} \sum_{\lambda \in \Lambda_{i}} \lambda_{i}^{2}(\xi)$.

Proof. Follows from (44).

Recall that the function $h(x)=\max _{a \in A} a(x)$ in $\mathbb{R}^{n}$ is called a support function of a compact convex body $A \subset \mathbb{R}^{n *}$.

Lemma 3. The function

$$
h_{i}(\xi)=\sqrt{F_{i}(\xi)}=\sqrt{\frac{1}{N_{i}} \sum_{\lambda \in \Lambda_{i}} \lambda_{i}^{2}(\xi)}
$$

is a support function of some ellipsoid ell $\left(\Lambda_{i}\right)$ in $\mathfrak{h}^{*}$. If the set $\Lambda_{i}$ is not contained in any proper subspace of $\mathfrak{h}^{*}$, then $\operatorname{dim} \operatorname{ell}\left(\Lambda_{i}\right)=n$.

Proof. For any nonnegative quadratic form $g$, it is true that the function $\sqrt{g}$ is a support function of some ellipsoid. If the form $g$ is non-degenerate, then this ellipsoid is full-dimensonal.

Theorem 1. It is true that

$$
\mathfrak{M}\left(\Lambda_{1}, \ldots, \Lambda_{n}\right)=n ! \operatorname{vol}\left(\operatorname{ell}\left(\Lambda_{1}\right), \ldots, \operatorname{ell}\left(\Lambda_{n}\right)\right),
$$

where in the measurement of the mixed volume it is assumed that the volume of the fundamental cube of the lattice $\mathbb{Z}^{n *}$ equals 1 .

Proof. For $X=T^{n}$ (taking into account the orthogonality of the action of $T^{n}$ on $\left.V\left(\Lambda_{i}\right)\right)$, the statement is easy to deduce from [AK, Tеорема 1].

Note, that the theorem 1 implies the following inequalities for the mean numbers of roots.

Corollary 1. For any supports $\Lambda_{1}, \ldots, \Lambda_{n}$ it is true that

$$
\begin{gathered}
\mathfrak{M}^{2}\left(\Lambda_{1}, \ldots, \Lambda_{n}\right) \geq \mathfrak{M}\left(\Lambda_{1}, \ldots, \Lambda_{n-1}, \Lambda_{n-1}\right) \cdot \mathfrak{M}\left(\Lambda_{1}, \ldots, \Lambda_{n}, \Lambda_{n}\right), \\
\mathfrak{M}^{n}\left(\Lambda_{1}, \ldots, \Lambda_{n}\right) \geq \mathfrak{M}\left(\Lambda_{1}\right) \cdot \ldots \cdot \mathfrak{M}\left(\Lambda_{n}\right),
\end{gathered}
$$

where, by definition, $\mathfrak{M}(\Lambda)=\mathfrak{M}(\Lambda, \ldots, \Lambda)$.

Proof. Follows from Alexandrov-Fenchel inequality (см. [Al]) for mixed volumes of ellipsoids ell $\left(\Lambda_{i}\right)$.

Remark 1. The inequalities for the numbers of roots from Corollary 1 are similarly to the Hodge inequalities for the intersection indices of projective algebraic surfaces. The connection between the Hodge inequalities and the Alexandrov-Fenchel inequalities was independently found by A. Khovansky and B. Teissier; see, for example, [KK]. 
3. Newton polytopes and ellipsoids. Recall that with a finite centrally symmetric set $\Lambda \subset \mathbb{Z}^{n *} \subset \mathfrak{h}^{*}$ we are considering two convex bodies: the Newton polytope $\operatorname{conv}(\Lambda)$ and ellipsoid ell $(\Lambda)$ with the support function

$$
h_{\Lambda}(\xi)=\sqrt{\frac{1}{N} \sum_{\lambda \in \Lambda} \lambda^{2}(\xi)},
$$

where $N$ is the number of elements of the set $\Lambda$.

Proposition 1. For almost all tuples $\left(P_{1}, \ldots, P_{n}\right)$ of real Laurent polynomials with supports $\Lambda_{1}, \ldots, \Lambda_{n}$ the number of solutions of the system $P_{1}=$ $\ldots=P_{n}=0$ is equal to $n ! \operatorname{vol}\left(\operatorname{conv}\left(\Lambda_{1}\right), \ldots, \operatorname{conv}\left(\Lambda_{n}\right)\right)$.

Proof. The set of such tuples $\left(P_{1}, \ldots, P_{n}\right)$ is an everywhere Zariski dense subset in the space of all tuples of Laurent polynomials with supports $\Lambda_{1}, \ldots, \Lambda_{n}$. Therefore, the statement is a consequence of Theorem BKK.

Lemma 4. $\operatorname{vol}(\operatorname{ell}(\Lambda)) \leq \operatorname{vol}(\operatorname{conv}(\Lambda))$

Proof. From Proposition 1 and from Theorem 11 it follows that the righthand side and the left-hand side of the formula are equal to the average number, respectively, of real and complex (i.e., all) roots of identical systems of equations.

This lemma is consistent with the following geometric statement.

Proposition 2. For any finite centrally symmetric $\Lambda$, it is true that $\operatorname{ell}(\Lambda) \subset$ $\operatorname{conv}(\Lambda)$.

Proof. For any finite set $\Lambda$ the support function of $\operatorname{conv}(\Lambda)$ at the point $\xi \in \mathfrak{h}$ is equal to $\max _{\lambda \in \Lambda} \lambda(\xi)$. If $\Lambda$ is centrally symmetric then $\max _{\lambda \in \Lambda} \lambda(\xi)=$ $\max _{\lambda \in \Lambda}|\lambda(\xi)|$. The support function of ellipsoid ell $(\Lambda)$ is equal to the root mean square of numbers $\{|\lambda(\xi)|: \lambda \in \Lambda\}$. The root mean square of a finite set of non-negative numbers does not exceed the maximum of them.

Theorem 2. Let real $\left(\Lambda_{1}, \ldots, \Lambda_{n}\right)$ be an average fraction of real roots of systems of trigonometric polynomials with supports $\Lambda_{1}, \ldots, \Lambda_{n}$. Then

$$
\operatorname{real}\left(\Lambda_{1}, \ldots, \Lambda_{n}\right)=\frac{\operatorname{vol}\left(\operatorname{ell}\left(\Lambda_{1}\right), \ldots, \operatorname{ell}\left(\Lambda_{n}\right)\right)}{\operatorname{vol}\left(\operatorname{conv}\left(\Lambda_{1}\right), \ldots, \operatorname{conv}\left(\Lambda_{n}\right)\right)}
$$

Proof. Follows from Theorem 1 and proposition 1 .

Example 1. In the case $n=1$, the segments ell $(\Lambda)$ and $\operatorname{conv}(\Lambda)$ coincide, if and if $\Lambda=\{\lambda,-\lambda\}$. From the Theorem 2 it follows that all zeros of any polynomial of the form $a z^{\lambda}+\bar{a} z^{-\lambda}$ belong to the unit circle, which is true, because these zeros are the $(2 \lambda)$-roots of $\bar{a} / a$.

Example 2. If $\Lambda=\{-\lambda, \ldots,-1,0,1, \ldots, \lambda\}$, then the ellipsoid ell $(\Lambda)$ is a segment

$$
\left[-\sqrt{\frac{\lambda(\lambda+1)}{3}}, \sqrt{\frac{\lambda(\lambda+1)}{3}}\right]
$$


Hence Theorem 2 implies that, for large $\lambda$, the average fraction of real zeros of trigonometric polynomials with support $\Lambda$ tends to $\sqrt{\frac{1}{3}} \simeq 0.577$.

4. Asymptotics of the average fraction of real roots. Let $\Delta \subset \mathfrak{b}^{*}$ be a centrally symmetric convex body, and $\Lambda=\Delta \cap \mathbb{Z}^{n *}$. Further, we assume that the following condition $(*)$ is satisfied

(*) if $\operatorname{dim} \Delta=k<n$, then $\Delta$ is contained in some $k$-dimensional subspace of $\mathfrak{h}^{*}$, generated by the vectors of the lattice $\mathbb{Z}^{n *}$.

For an integer $m>0$ we put $\Delta_{m}=m \Delta, \Lambda_{m}=\Delta_{m} \cap \mathbb{Z}^{n *}, N_{\Lambda, m}=\# \Lambda_{m}$. Recall that a function $h_{\Lambda}=\sqrt{F_{\Lambda}}$, where

$$
F_{\Lambda}(\xi)=\frac{1}{N_{\Lambda}} \sum_{\lambda \in \Lambda} \lambda^{2}(\xi)
$$

is the support function of ellipsoid $\operatorname{ell}(\Lambda) \subset \mathfrak{h}^{*}$.

Lemma 5. For $m \rightarrow \infty$, the sequence of functions $\frac{1}{m^{2}} F_{\Lambda, m}$ converges locally uniformly to some quadratic form $F_{\Delta}$.

Proof. Choose a basis of the lattice $\mathbb{Z}^{n *}$ and declare it an orthonormal basis of the space $\mathfrak{h}^{*}$. Using the appropriate metric, we identify the spaces $\mathfrak{h}$ and $\mathfrak{h}^{*}$, as well as integer lattices $\mathbb{Z}^{n} \subset \mathfrak{h}$ and $\mathbb{Z}^{n *} \subset \mathfrak{h}^{*}$. Let $\operatorname{dim} \Delta=k$. Then

$$
\frac{1}{m^{2}} F_{\Lambda, m}(\xi)=\frac{1}{m^{2} N_{\Lambda, m}} \sum_{\lambda \in \Lambda_{m}}\langle\lambda, \xi\rangle^{2}=\frac{m^{k}}{N_{\Lambda, m}} \sum_{\alpha \in \frac{\Lambda_{m}}{m}}\langle\alpha, \xi\rangle^{2} \frac{1}{m^{k}} .
$$

Now notice that $N_{\Lambda, m} \asymp \operatorname{vol}_{k}(\Delta) m^{k}$, and $\sum_{\alpha \in \frac{\Lambda_{m}}{m}}\langle\alpha, \xi\rangle^{2} \frac{1}{m^{k}}$ is the integral sum for an integral of the function $f(x)=\langle x, \xi\rangle^{2}$ over the polytope $\Delta$. Hence

$$
\frac{1}{m^{2}} F_{\Lambda, m}(\xi) \rightarrow F_{\Delta}(\xi)=\frac{1}{\operatorname{vol}_{k}(\Delta)} \int_{\Delta}\langle x, \xi\rangle^{2} d x
$$

Corollary 2. Let $m \rightarrow \infty$. Then

(1) the sequence of functions $\frac{1}{m} h_{\Lambda, m}$ converges locally uniformly to the support function $h_{\Delta}$ of some ellipsoid $\operatorname{ell}(\Delta)$

(2) the sequence of ellipsoids $\frac{1}{m} \operatorname{ell}\left(\Lambda_{m}\right)$ converges in the Hausdorff topology to the ellipsoid ell $(\Delta)$.

Proof. Since, by definition, $h_{\Delta}=\sqrt{F_{\Delta}}$, then both statements are direct consequences of Lemma 5.

Lemma 6. If the condition $\left(^{*}\right)$ is satisfied for the convex body $\Delta$, then for $m \rightarrow \infty$ the sequence of convex polytopes $\frac{1}{m} \operatorname{conv}\left(\Lambda_{m}\right)$ converges in Hausdorff topology to the convex body $\Delta$.

Proof. This follows from the definition of the set $\Lambda_{m}$. 
Theorem 3. Let $\Delta_{1}, \ldots, \Delta_{n}$ be convex bodies in the space $\mathfrak{h}^{*}$, satisfying the condition $(*)$, and $\Lambda_{i}=\Delta_{i} \cap \mathbb{Z}^{n *}$. Then

$$
\lim _{\inf \left(m_{1}, \ldots, m_{n}\right) \rightarrow \infty} \operatorname{real}_{n}\left(\left(\Lambda_{1}\right)_{m_{1}}, \ldots,\left(\Lambda_{n}\right)_{m_{n}}\right)=\frac{\operatorname{vol}\left(\operatorname{ell}\left(\Delta_{1}\right), \ldots, \operatorname{ell}\left(\Delta_{n}\right)\right)}{\operatorname{vol}\left(\Delta_{1}, \ldots, \Delta_{n}\right)}
$$

Proof. Let $\left(m_{1}\right)_{i}, \ldots,\left(m_{n}\right)_{i}$ be an increasing sequence of tuples of positive integers, and let $\left(\Lambda_{1}\right)_{\left(m_{1}\right)_{i}}, \ldots,\left(\Lambda_{n}\right)_{\left(m_{n}\right)_{i}}$ be the corresponding sequence of tuples of supports. Then the passage to the limit based on the corollary 2 (2) and the lemma 6, gives the required statement.

Corollary 3. Let $\alpha_{1}, \ldots, \alpha_{n}$ be positive numbers. Then, when replacing $\Delta_{i} \rightarrow \alpha_{i} \Delta_{i}$ (with such replacements the condition $\left(^{*}\right)$ remains satisfied) the asymptotic behavior of $\operatorname{real}_{n}\left(\Lambda_{m_{1}}, \ldots, \Lambda_{m_{n}}\right)$ is preserved.

Proof. From (6) it follows, что $h_{\alpha \Delta_{i}}=\alpha h_{\Delta_{i}}$. Therefore, the numerator and denominator of a fraction in right-hand side (7) are multiplied by $\alpha_{1} \cdots \alpha_{n}$.

\section{An example of calculating the asymptotics of the average frac-} tion of real roots. Here we calculate the limit

$$
\lim _{\inf \left(m_{1}, \ldots, m_{n}\right) \rightarrow \infty} \operatorname{real}_{n}\left(\Lambda_{m_{1}}, \ldots, \Lambda_{m_{n}}\right)
$$

for balls $\Delta_{i}$ radii $R_{i}$.

Theorem 4. It is true that

$$
\lim _{\inf \left(m_{1}, \ldots, m_{n}\right) \rightarrow \infty} \operatorname{real}_{n}\left(m_{1}, \ldots, m_{n}\right)=\left(\frac{\beta_{n}}{\sigma_{n}}\right)^{\frac{n}{2}},
$$

where $\beta_{n}=\int_{-1}^{1} x^{2}\left(1-x^{2}\right)^{\frac{n-1}{2}} d x$, and $\sigma_{n}$ is a volume of $n$-dimensional ball of radius 1 .

We give the values of $\beta_{n}$ for $n \leq 20$ (if $n=1$, then $\sqrt{\frac{1}{2} \beta_{1}}=\frac{1}{\sqrt{3}}$; see Example 2).

\begin{tabular}{r|ccccccccccc}
$n$ & 1 & 2 & 3 & 4 & 5 & 6 & 7 & 8 & 9 & 10 \\
$\beta_{n}$ & $\frac{2}{3}$ & $\frac{\pi}{8}$ & $\frac{4}{15}$ & $\frac{\pi}{16}$ & $\frac{16}{105}$ & $\frac{5 \pi}{128}$ & $\frac{32}{315}$ & $\frac{7 \pi}{256}$ & $\frac{256}{3465}$ & $\frac{21 \pi}{1024}$ \\
\hline$n$ & 11 & 12 & 13 & 14 & 15 & 16 & 17 & 18 & 19 & 20 \\
$\beta_{n}$ & $\frac{512}{9009}$ & $\frac{33 \pi}{2048}$ & $\frac{4096}{109395}$ & $\frac{429 \pi}{32768}$ & $\frac{2048}{45045}$ & $\frac{715 \pi}{65536}$ & $\frac{65536}{2078505}$ & $\frac{2431 \pi}{262144}$ & $\frac{131072}{4849845}$ & $\frac{4199 \pi}{524288}$
\end{tabular}

Remark 2. The expression $x^{2}\left(1-x^{2}\right)^{\frac{n-1}{2}} d x$ is the so-called differential Tchebichef binomial. Tchebichef proved [Ch] that the binomial $x^{m}\left(a+b x^{n}\right)^{p} d x$ is non-integrable outside three (known to L. Euler) cases. For odd $n$, the above expression refers to the first one, and for even $n$ - to the third case.

Application of Lemma 6 and Theorem 3 reduces the proof of Theorem 4 to the following statement. 
Proposition 3. It is true that $\operatorname{ell}\left(\Delta_{i}\right)=\sqrt{\frac{\beta_{n}}{\sigma_{n}}} \Delta_{i}$.

Proof. According to Corollary 2, the sequence of ellipsoids $\frac{1}{m}$ ell $\left(\left(\Lambda_{i}\right)_{m}\right)$ converges to the ellipsoid ell $\left(\Delta_{i}\right)$ with support function

$$
h_{\Delta_{i}}(\xi)=\sqrt{\frac{1}{\operatorname{vol}_{k}\left(\Delta_{i}\right)} \int_{\Delta_{i}}\langle x, \xi\rangle^{2} d x} .
$$

The right side of the equation, as it is easy to see, is equal to $|\xi| R_{i} \sqrt{\frac{\beta_{n}}{\sigma_{n}}}$, where $R_{i}$ is the radius of the ball $\Delta_{i}$. Thus, $h_{\Delta_{i}}(\xi)$ is the support function of the ball of radius $R_{i} \sqrt{\frac{\beta_{n}}{\sigma_{n}}}$ centered at the point 0 . This ball is $\sqrt{\frac{\beta_{n}}{\sigma_{n}}} \Delta_{i}$. The proposition is proved.

\section{REFERENCES}

[ADG] Jürgen Angst, Federico Dalmao and Guillaume Poly. On the real zeros of random trigonometric polynomials with dependent coefficients. Proc. Amer. Math. Soc. (147:1), 2019, 205-214 (arXiv:1706.01654)

[Ka] M. Kac. On the average number of real roots of a random algebraic equation. Bull. Amer. Math. Soc. 49 (1943), 314-320 (Correction: Bull. Amer. Math. Soc., Volume 49, Number 12 (1943), 938-938)

[EK] A. Edelman, E. Kostlan. How many zeros of a real random polynomial are real? Bull. Amer. Math. Soc. 32, no.1 (1995), 1-37 (arXiv:math/9501224)

[AK] D. Akhiezer, B. Kazarnovskii. Average number of zeros and mixed symplectic volume of Finsler sets. Geom. Funct. Anal., 2018, (28:6), 1517-1547.

[ZK] D. Zaporozhets, Z. Kabluchko. Random determinants, mixed volumes of ellipsoids, and zeros of Gaussian random fields. Journal of Math. Sci., vol. 199, no.2 (2014), pp. $168-173$

[Al] A. D. Aleksandrov. To the theory of mixed volumes of convex bodies. Part II: New inequalities for mixed volumes and their applications, in: Selected Works, Part I, ed. by Yu.G.Reshetnyak and S.S.Kutetaladze, Amsterdam, Gordon and Breach, 1996 (English translation).

[KK] K. Kaveh, A. G. Khovanskii. Newton-Okounkov bodies, semigroups of integral points, graded algebras and intersection theory. Ann. of Math., (176:2), 2012, 925-978.

[B] D. N. Bernstein. The number of roots of a system of equations. Funct. Anal. Appl. (9:3), 1975, 183-185

[Ch] P. Tchebichef. Sur l'intégration des différentielles irrationnelles. Journal de mathématiques pures et appliquées. 1853. Vol. XVIII. 87-111.

Institute for Information Transmission Problems, Moscow kazbori@gmail.com. 